\title{
PREDICTIVE MAINTENANCE OF SINGLE PHASE AC MOTOR USING IOT SENSOR DATA AND MACHINE LEARNING (SIMPLE LINEAR REGRESSION AND MULTIPLE LINEAR REGRESSION ALGORITHMS)
}

\author{
Jakra A. Husain \\ Department of Electronics and Communication, \\ Ballarpur Institute of Technology Ballarpur, India
}

Abstract-Predictive maintenance techniques are proposed to find out the condition of operating equipment in order to predict when failure will take place or maintenance will be essential. The crucial aim is to provide cost savings over schedule based preventative maintenance or unexpected reactive maintenance, which could result in machinery being unavailable throughout the critical periods. There are many approaches for predicting failures, in this project current analysis approach is used where the voltage and current of electricity is measured which is supplied to the motor. But these analyses can't situate by themselves. Architecture is desirable to sustain the end-to-end workflow that allows for processing at the edge and moves the data from the integrated network to a central depository for analysis and monitoring. To do predictive maintenance, firstly we have to build an integrated network of PZEM-004T meter with a Node MCU ESP8266 -12E Module that will monitor and collect data about the voltage, current, and active power of $A C$ motor under test which are used here as key variables for determining the status of AC motor. Our approach consists of two stages. The first incorporates data gathering, data processing, and then applying supervised learning methods to gain insights from the data and the second follows the first to build predictive models which can work easily and efficiently as per our requirement. Using Regression ML (Machine Learning) an algorithm that is Simple Linear Regression (SLR) And Multiple Linear Regression (MLR) machine under test (motor 1 and motor 2) performance can be optimize and provide new statistic patterns which make the backbone of prediction analysis. The machine learning algorithm is applied to the collected and processed data based on which results are obtained. The obtained test result is compared with obtained predicted result based on which the prediction accuracy of each machine learning algorithm is decided. Accuracy can be further improved by using much more data while applying the machine learning algorithms, as the number of data streams increases prediction accuracy

\author{
Prof. Ashish Manusmare \\ Department of Electronics and Communication, \\ Ballarpur Institute of Technology Ballarpur, India
}

increases. These models are trained for long time to respond to new data or new values and then it delivers the results we need with more accuracy.

Keywords: IoT, Machine Learning, SLR, MLR

\section{INTRODUCTION}

Predictive modeling is the general concept of building a model that is capable of making predictions. Typically, such a model includes a machine learning algorithm (SLR or MLR) that learns certain properties from a training dataset in order to make those predictions. Predictive modeling can be divided further into two sub areas: Regression and classification. Regression models are based on the analysis of relationships between variables and trends in order to make predictions about continuous variables, e.g. the prediction of abnormal key variables of motor 1 and motor 2 under test the prediction of motors abnormal working due to heavy load which leads to excess power consumption ,the prediction of timestamp needed for machine maintenance. In contrast to regression models, the task of pattern classification is to assign discrete class labels to particular observations as outcomes of a prediction._Machine learning and predictive analytics [2] go hand in hand as prediction models have the distinctive qualities which include a machine learning algorithm. These models are trained for long time to respond to new data and then delivers the result we need.Predictive maintenance predicts failure, and the actions could include corrective actions, the replacement of system, or even planned failure. This can lead to major cost savings, higher predictability, and the increased availability of the systems. Predictive maintenance avoids or minimizes the downtimes. This will avoid an unhappy customer, save money, and sometimes save lives. Optimize the periodic maintenance operations. The industrial Internet of Things (IIoT) is the use of Internet of Things (IoT) technologies in industries which exploit the 


\section{International Journal of Engineering Applied Sciences and Technology, 2019 Vol. 4, Issue 4, ISSN No. 2455-2143, Pages 128-135 \\ Published Online August 2019 in IJEAST (http://www.ijeast.com)}

machine data generated by various sensors used and applies various analytics on it to gain useful information. The use of Machine Learning thus proves a vital component in IIoT having use cases in quality management and quality control, lowering the cost of maintenance and improving the overall manufacturing process. ML algorithms are loosely classified into 3 divisions unsupervised learning, supervised learning and reinforcement learning. Machine learning like data mining has evolved significantly in recent years. Machine learning like Data mining aim at analyzing the complete data and try to find sensible patterns in it. On the other hand, in option of obtaining information for real world understanding as is that the case in data processing applications; machine learning make use of the information to spot patterns in data and improve program actions and model if build so. The basic approach in Machine Learning especially in supervised machine learning is that the aim of inferring a importance from label on the data used for training which consists of set of training samples.

Predictive maintenance for industry is a method of preventing asset failure by analyzing production data to identify patterns and predict issues before they happen. Until now, industry managers and machine operators carried out scheduled maintenance and regularly repaired machine parts to prevent downtime. In addition to consuming unnecessary resources and driving productivity losses. Predictive Maintenance has quickly emerged as a leading Industry use case for manufacturers and asset managers. An implementing industrial IoT technology to monitor asset health, optimize maintenance schedules, and gaining real-time alerts to operational risks, allows manufacturers to lower service costs, maximize uptime, and improve production throughput. Predictive analytics are applied to the machine data in order to predict conditions of upcoming failure. A dashboard for predictive analytics synthesizes operational data, allowing process and maintenance engineers to address actionable insights in the form of corrective action. Manufacturers and their customers get a range of business benefits from predictive maintenance. In case of supervised learning, each example may be a base which contains an input object (which generally is represented here as values regarding various parameters of motors or as a vector) and the output has a value act as a signal to supervise the model. A supervised learning rule initially performs the examination task from the sample data and constructs a provisional function, so as to map new input data. A optimum setting in all probability aids the rule to precisely mark the category labels for enclosed instances and therefore the same requirements the supervised learning rule to cut back from the coaching information to enclosed things in a very proper manner. The supervised learning algorithms are used in various application areas such as predictive maintenance of machines in industries as in this case predictive maintenance of AC motor, fraud detection, finance, producing, testing, weather prediction, and so on .Predictive maintenance avoids both the limits and maximizes the use of its resources. Predictive maintenance will detect the anomalies and malfunction patterns and provide early warnings. These warnings can enable efficient maintenance of those components or device used.

\section{LITERATURE SURVEY}

Junmin park et al.,2018 proposed data compression and prediction using machine learning algorithms for industrial IoT. They presented several techniques for compressing and predicting huge amounts of industrial data. Industrial IoT generates huge data that is useful for getting future prediction from data analysis but storing all the data is a burden. To resolve this issue, they proposed a system to compress the industrial data using neural network regression technique into a representative vector with lossy compression. For efficiency of the compression, they use divide-and-conquer method such that the industrial data can be handled by the chunk size of data. The neural network regression technique is not enough to compress the industrial data generated on an hourly basis. So they compress the data using the divide-and-conquer method. They divide the data generated by the divide process into time units, and apply neural regression to each of them. In conquer, there is application of various machine learning techniques and they choose the method with the highest accuracy. As a result, they claim that it is an efficient data compression method to represent data by linear regression, using divide and conquer method, divide data by time, and use machine learning algorithm using Euclidean Distance in conquer process. The disadvantage of this proposed method is that loss compression cannot be recovered as original data, it is expected that future data is predictable by expressing industrial data as a single equation through this process and utilizing the tendency.[3]

In 2017 Ameeth kanawaday and Aditya sane proposed a methodology for predictive maintenance of industrial machines using IoT sensors data and machine learning technique. The industrial Internet of Things (IIoT) is the use of Internet of Things (IoT) technologies in manufacturing which uses the machine data generated by various sensors attached to the respective machine under test and applies different analytics on it to achieve useful information. The data collected by the industrial machines under test is usually accompanied by a date time component which proves essential for predictive modeling. This paper explores the use of Auto Regressive Integrated Moving Average (ARIMA) forecasting on the time series data collected from various sensors from a Slitting Machine, to predict the possible future failures and quality defects, thus improving the overall manufacturing and maintenance process. The use of Machine Learning thus proves a essential component in Industrial IoT in quality management, quality control and predictive maintenance, reducing the cost of maintenance, improving the overall manufacturing process and useful in predicting the 


\section{International Journal of Engineering Applied Sciences and Technology, 2019 Vol. 4, Issue 4, ISSN No. 2455-2143, Pages 128-135 \\ Published Online August 2019 in IJEAST (http://www.ijeast.com)}

maintenance time. If this model predicts the cycle as a bad production cycle, necessary steps must be taken to avoid it from happening this is the serious issue of this methodology. [4]

Joseph siryani et al., 2017 presented a framework for a decision-support system (DSS) that operates within the Internet of Thing ecosystem. The objective of their paper is to propose a predictive analytics model for ESM network operations decision support. The model employs a Bayesian network and three machine learning (ML) predictive models with Naïve Bayes (NB), Decision Tree (DT) and Random Forest (RF), that will improve ESM systems predictions about whether to send a technician to a customer location to resolve an ESM fault. Therefore, it can reduce travel expense costs during the ESM network operations and maintenance (O\&M) stage by deploying maintenance only where and when it is needed. They demonstrate the efficiency of their approach with a complete Bayesian Network machine learning classifiers: Naïve Bayes, Random Forest and Decision Tree. Results demonstrate that their approach generates statistically important estimations and that the DSS will improve the cost effectiveness of ESM network operations and maintenance. The random forest, among the various predictive models, is an important technique for the analysis of smart meter data for large datasets, with the highest accuracy of $96.69 \%$, ensuring the highest operational savings, other machine learning algorithm like NB, DT and BN does not provide that much accuracy as compared to random forest technique.

\section{PROPOSED WORK}

\section{Proposed Methodology}

Predictive Maintenance determination of machine (single phase AC motor), its proper working, specifications and its remaining useful life before failure depends upon the type of hardware and Machine learning algorithm used.

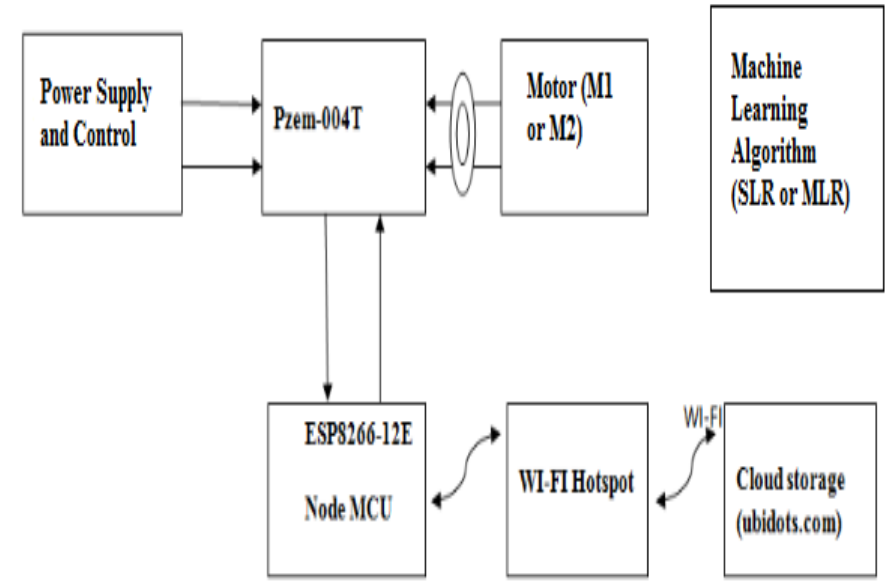

Figure: 3.1 Block Diagram of the Existing System.

\section{Block Diagram Explanation}

In this existing system motors are used for test, here current, voltage and power consumption are the key variables the data about this key variables is measured with the help of Pzem004T.The Pzem-004T used here will measure large numbers (upto 5000 Points)of different values .Pzem-004T attached to the motor only become meaningful when predictions and its control is done in response to huge data .The Pzem-004T device sends the data when the machine shows a change in state which usually is sampled per second. Sensor data is not passed directly to the cloud it is done using a gateway.

Thus the outcome of Pzem-004T device attached to motor is fed to Esp8266-12E WI-FI Module Node MCU it also act as an industrial gateway. The Esp8266-12E Node MCU for the machine stores data for various parameters which need to be monitored. Data generated from the machine was collected using Pzem-004T and pushed to the Industrial cloud storage using industrial gateway (Esp8266-12E WI-FI Module Node MCU ).This obtained data need to be sampled per second and should be collected for a period of one month approximately. Once the data from Pzem-004T device enters the cloud storage part it gets lands on a streaming data processor. The purpose of this is to allow continuous flow of sensor data and efficiently transfer data stream to a data storage unit -a data lake. A data lake stores the data collected by Pzem-004T device equipped at motors. Number of sets of Pzem-004T device readings which is measured at a corresponding time is presented. when the information is needed it gets loaded into big data warehouse. The big data warehouse consist of cleaned structured collected data, it contains key variables related to motors, parameters which cause failure of motors and also it contains context information regarding motor type, motors location and motors replacement date.

After the preparation of this all data, it is analyzed with machine learning algorithm. During the data analysis stage various forms of technical assumptions are considered which help to select the best fit ML algorithms. Here Simple Linear Regression (SLR) and Multiple Linear Regression (MLR) Supervised Machine Learning Algorithm are used.ML algorithms reveals the hidden correlations in sets of data for detecting data patterns which are abnormal. This pattern of data are reflected in models which is termed as prediction models. After this the prediction models are built, training is provided to them and then they are used to identify the current, voltage and power consumption conditions, normal and abnormal working of motors, and also it is helpful to estimate the useful life of motors under test. The prediction models needs training for long time to respond to new data and then delivers the outcome we need.

\section{Machine Learning Techniques Used in the Project (Supervised Machine Learning)}

Machine Learning focuses on prediction and can make data analysis and prediction efficient by looking at huge amounts of data simultaneously. It monitors accuracy over statistical 


\section{International Journal of Engineering Applied Sciences and Technology, 2019 Vol. 4, Issue 4, ISSN No. 2455-2143, Pages 128-135 \\ Published Online August 2019 in IJEAST (http://www.ijeast.com)}

importance. The majority of practical machine learning technique uses supervised learning. In supervised Machine Learning which is used in this existing system all data is labeled and the algorithms learns to predict the outcome from the input data .Supervised learning is where we have input variable $(\mathrm{X})$ and an output variable $(\mathrm{Y})$ and we use an algorithm to learn the mapping function from the input to the output. The basic representation can be given in the form of expression below which shows that the output variable (Y) is the function of input variable $(\mathrm{X})$.

$$
\mathrm{Y}=\mathrm{f}(\mathrm{X})
$$

The aim in supervised learning is to approximate the mapping function so well that when we have new input data $(X)$ then we can predict the output variables (Y) for that data. It is called supervised learning because the process of algorithm learning from the training datasets can be considered as a teacher supervising the learning process. We know the correct answers; the algorithm iteratively makes predictions on the training data and is corrected by the teacher. Learning process stops when the algorithm achieves an acceptable level of performance.

\section{Simple Linear Regression for Regression problems}

Linear Regression is a machine learning algorithm based on supervised learning. It performs a regression task. Regression models a target prediction value based on independent variables. It is mostly used for finding out the relationship between variables and forecasting. Different regression models differ based on the kind of relationship between dependent and independent variables they are considering and the number of independent variables or key parameters being used. Linear Regression is used in troubles where the label is of continuous nature e.g. Sales of a retail chain, continuous monitoring of any machine in terms of specific parameter related to that machine. Such algorithms are mostly used to for decision-making process to solve problems like if it is necessary to replace machine or not, is there any need of maintenance or not, monitoring of machine in industries at particular interval of time is necessary or not, is it working normally or abnormally due to heavy load and many more.

\section{Multiple Linear Regressions}

Multiple Linear Regression attempts to build the Relationship between two or more features or key variables and a response by fitting a linear equation to observed data. The steps to perform multiple linear Regression are almost similar to that of simple linear Regression. The dissimilarity lies in the evalution. We can use it to find out which key variable has the highest impact on the predicted outcome and we can also eliminate the key variable or factor which has a lowest impact on predicted outcome and which has not much contribution to the accuracy of prediction. Multiple linear regression attempts to model the relationship between two or more descriptive variables and a response variable by fitting a linear equation to observed data. Every value of the independent variable $\mathrm{x}$ is related with a value of the dependent variable $y$. It is the most regular form of Linear Regression.

Workflow Diagram for Supervised Machine Learning In the subsequent section, major steps of a typical supervised learning task are given, and the diagram below gives us an obvious understanding of how they are associated.

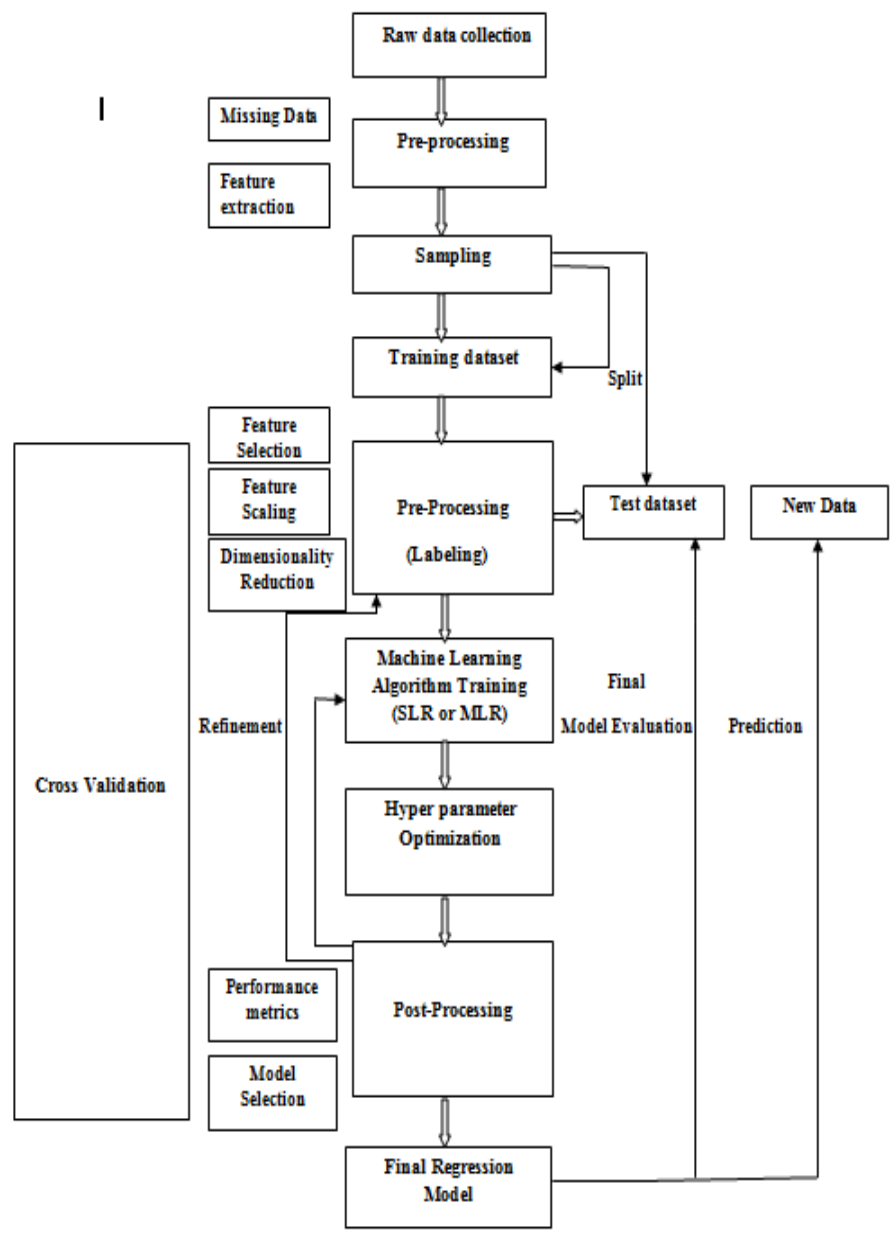

Figure 3.2: Flowchart for Supervised Machine Learning.

\section{RESULT}

For developing and managing a production-ready model, the work has undergone the following stages.

\section{Step 1: Data Gathering}

This step is very important because the quality and the quantity of data that has been gathered will directly determine how good is the prediction model .The process of gathering the data depends upon the application for which the data is been collected. Here we are building a project for predictive maintenance of industrial machine hence it is necessary to collect the real time data. Gathering data is the most important 


\section{International Journal of Engineering Applied Sciences and Technology, 2019 \\ Vol. 4, Issue 4, ISSN No. 2455-2143, Pages 128-135 \\ Published Online August 2019 in IJEAST (http://www.ijeast.com)}

step in solving any supervised machine learning problem. Your text classifier can only be as good as the dataset it is built from. The more training examples we have, it is better. This will help our model generalize better. Building and training a model is only one part of the workflow. Understanding the characteristics of your data beforehand will enable you to build a better model. This could simply mean obtaining a higher accuracy. It could also mean requiring less data for training, or fewer computational resources. Data is at the core of any Machine Learning problem. All the strides being made using Machine Learning these days would not be possible if not for access to relevant data. Luckily, we live in a time where an abundance of data is available on the web; all we need are the skills to identify and extract meaningful datasets. So let's get started to see what it takes to identify, scrape and build a good-quality Machine Learning dataset.

Firstly an integrated system of Pzem-004T with Node MCU ESP8266 using Ubidots IoT firmware is built the main component for data collection used here is Pzem-004T meter which gives the corresponding current, voltage and power consumption reading of ac motor which is used here as an industrial machine. This meter collects the data which is numeric in form and display it in dashboard of Ubidots explorer app, but the collected data cannot be used directly for performing the analysis process as there might be a lot of missing data, extremely large values, unorganized text data or noisy data. Therefore, to solve this problem Data Preparation and data Processing is done.

\begin{tabular}{llll} 
M1 Voltage, M1 Current, M1 Power & -1 & -1 \\
\hline June 152019 at $20: 50: 24$ & 230.5 & -1 & 60 \\
\hline June 152019 at 20:50:21 & 230.5 & 0.26 & 61 \\
\hline June 152019 at 20:50:19 & 230.5 & 0.26 & 62 \\
\hline June 152019 at 20:50:17 & 230.5 & 0.26 & 62 \\
\hline June 152019 at 20:50:15 & 230.5 & 0.26 & 61 \\
\hline June 152019 at 20:50:13 & 230.3 & 0.26 & 61 \\
\hline June 152019 at 20:50:11 & 230.3 & 0.26 & 60 \\
\hline June 152019 at 20:50:08 & 230 & 0.26 & 62 \\
\hline June 152019 at 20:50:06 & 229.8 & 0.26 & 61 \\
\hline June 152019 at 20:50:04 & 229.8 & 0.26 & 60 \\
\hline June 152019 at 20:50:02 & 229.89999 & 0.26 & \\
\hline
\end{tabular}

Figure 4.1 : Data Collected for Motor 1 on June 152019

Figure 4.1 gives us a clear understanding of how data is measured by Pzem-004T meter, which is stored into ESP8266 Node MCU Module. This module acts as a WIFI device for sending data to IoT firmware Ubidots. Thus ubidots display this data to us in such a format which is shown in figure 4.1 5.1.1.Here the date and time of measurement is indicated clearly, which shows the respective data about voltage, current and power is measured on respective date at respective time. For e.g on june 152019 at 20:50:21 the corresponding measured value of voltage was 230.5 volts, value of current was 0.26 Amps and respective power at this time was 60 Watt.
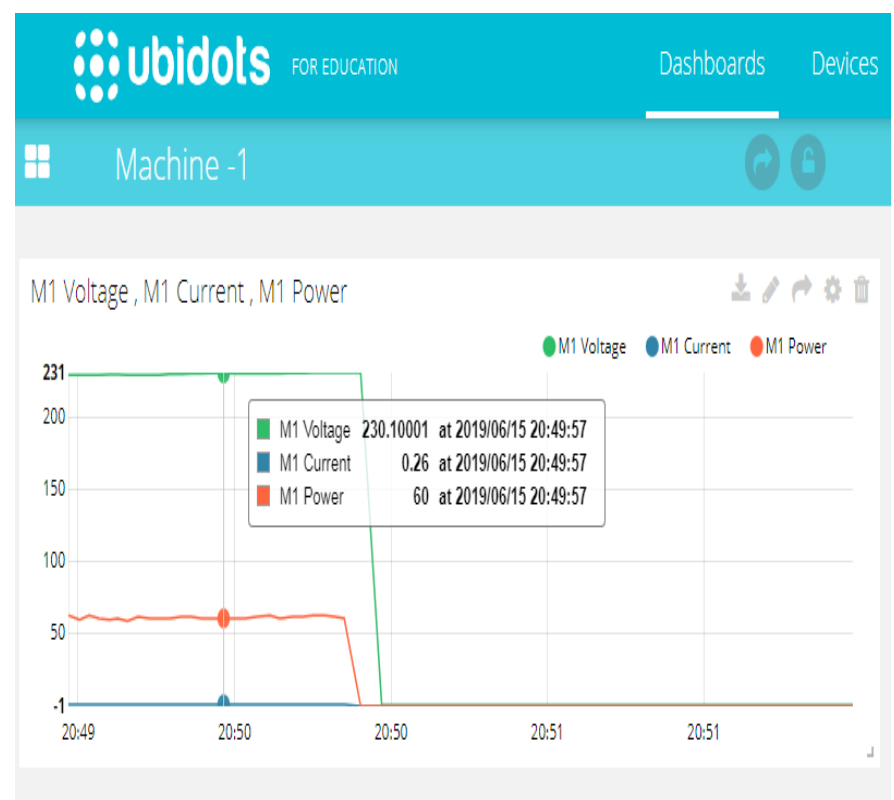

Time (in hh:mm:ss)

Figure 4.2: Graph of Collected Data (Current, Voltage And Power) for Motor 1.

Figure 4.2 Shows the graph of obtained readings (voltage, current and power) for motor1. For example at time 20:49:57 the readings for voltage, current and power was obtained as 230.10001 V, 0.26 Amp and 60 Watts respectively.

\section{Step 2: Data Preparation and Data Processing}

The gathered data is loaded into a suitable place (downloading the data which is in the form of. csv file and this is displayed in the excel sheet) and further it is prepared for using it in our ML training .Here all data is kept together and then it is randomize. Randomization is necessary because we don't want that the order of our data affect how we learn, since that not the part of determining whether predictive maintenance is required or not. At this point, we have assembled our dataset and gained insights into the key characteristics of our data. In other word we want to determine maintenance time and failure of ac motor which should be independent of the sequence of current and voltage falls value below threshold it means whatever the low threshold values comes first it should determine correctly. 


\begin{tabular}{|r|r|r|r|r|}
\hline A & \multicolumn{1}{|c|}{ B } & \multicolumn{1}{c|}{ C } & \multicolumn{1}{c|}{ D } & \multicolumn{1}{c|}{ E } \\
\hline $1.56 \mathrm{E}+12$ & $04 / 06 / 201918: 08$ & 0 & -1 & -1 \\
\hline $1.56 \mathrm{E}+12$ & $04 / 06 / 201918: 08$ & 233.10001 & 0.2 & -1 \\
\hline $1.56 \mathrm{E}+12$ & $04 / 06 / 201918: 08$ & 233.10001 & 0.27 & 63 \\
\hline $1.56 \mathrm{E}+12$ & $04 / 06 / 201918: 08$ & 233.10001 & 0.27 & 62 \\
\hline $1.56 \mathrm{E}+12$ & $04 / 06 / 201918: 08$ & 233.10001 & 0.27 & 61 \\
\hline $1.56 \mathrm{E}+12$ & $04 / 06 / 201918: 08$ & 231.8 & 0.27 & 61 \\
\hline $1.56 \mathrm{E}+12$ & $04 / 06 / 201918: 08$ & 231.8 & 0.26 & 62 \\
\hline $1.56 \mathrm{E}+12$ & $04 / 06 / 201918: 08$ & 231.7 & 0.26 & 61 \\
\hline $1.56 \mathrm{E}+12$ & $04 / 06 / 201918: 08$ & 231.7 & 0.27 & 61 \\
\hline $1.56 \mathrm{E}+12$ & $04 / 06 / 201918: 08$ & 232.89999 & 0.27 & 62 \\
\hline $1.56 \mathrm{E}+12$ & $04 / 06 / 201918: 08$ & 232.89999 & 0.26 & 62 \\
\hline $1.56 \mathrm{E}+12$ & $04 / 06 / 201918: 08$ & 233.39999 & 0.26 & 62 \\
\hline $1.56 \mathrm{E}+12$ & $04 / 06 / 201918: 08$ & 233.39999 & 0.27 & 60 \\
\hline $1.56 \mathrm{E}+12$ & $04 / 06 / 201918: 07$ & 233.10001 & 0.27 & 62 \\
\hline $1.56 \mathrm{E}+12$ & $04 / 06 / 201918: 07$ & 233.10001 & 0.27 & 62 \\
\hline $1.56 \mathrm{E}+12$ & $04 / 06 / 201918: 07$ & 233.10001 & 0.27 & 63 \\
\hline $1.56 \mathrm{E}+12$ & $04 / 06 / 201918: 07$ & 233.10001 & 0.3 & 63 \\
\hline $1.56 \mathrm{E}+12$ & $04 / 06 / 201918: 07$ & 233.2 & 0.3 & 72 \\
\hline $1.56 \mathrm{E}+12$ & $04 / 06 / 201918: 07$ & 233.2 & 0.3 & 70 \\
\hline $1.56 \mathrm{E}+12$ & $04 / 06 / 201918: 07$ & 233.39999 & 0.3 & 73 \\
\hline $1.56 \mathrm{E}+12$ & $04 / 06 / 201918: 07$ & 233.39999 & 0.26 & 73 \\
\hline $1.56 \mathrm{E}+12$ & $04 / 06 / 201918: 07$ & 233.5 & 0.26 & 62 \\
\hline & & & & \\
\hline
\end{tabular}

Figure 4.3: M1 Voltage,M1 Current And M1 Power .Csv File In Excel Sheet.

Figure 4.3 shows the list of collected dataset in excel sheet which is collected on June 042019 at 18:07 to 18:08 related to voltage, power and current of Motor 1 . The gathered data is loaded into a suitable place (downloading the file from Ubidots which is in the form of. csv file and opening it into excel sheet, which is displayed in the excel sheet in the format as shown in figure 4.3) and further it is prepared for using it in our ML training. It gives us a clear representation of corresponding Voltage, Current and Power at corresponding date and time. For example the value of voltage at 18:07 is 233.39999 Volts at this time the corresponding value of current and power is $0.26 \mathrm{Amps}$ and 73 Watts respectively.

\section{Step 3: Choosing a Model.}

Developing the model using well-known ML techniques or by defining new operations and approaches.

There are many models that researchers and data scientists have created over the years. Some are very well suited for image data, others for sequences such as text or music, some for numerical data and other for text based data. In this existing system supervised model is used. The majority of realistic machine learning uses supervised learning. In supervised Machine Learning technique all the data is labeled and the algorithms learns to predict the outcome from the input data. Supervised learning technique is where we have input variables (X) and an output variable (Y) and we utilize an algorithm to learn the mapping function from the input to the output. The purpose is to fairly accurate the mapping function so well that when we have new input data $(x)$ then the system can predict the output variables (Y) for that new data.
Step 4: Training, Evaluating and Tuning Proposed Model Based on Gathered and Processed Data.

In this step we will use our data to incrementally improve our models ability to predict. The values we have available with us to adjust or train the model are just key variables of motor that is current, voltage and power. there is no other way to affect the training of model. The training process involves initializing some random values for key variables and attempting to predict the output with those values. we can compare the model predictions with the output it should have produced and adjust the value in pre-processing step, such that we will have more accurate predictions on the next time around. So this process repeats and each iteration or cycles of updating the data is called one training step. Evaluation allows us to test our model against data that has never been used for training. This is meant to be representative of how the model might perform in the real world. Once evaluation is completed it is possible that we can further improve our training in anyway if necessary. Once training is done guided by the Evaluation step, it's finally time to use our model to do something useful.

Table 1: Table for Representation of Various Conditions of Motor1 and Motor2 based on which the Machine learns. (Here Voltage is measured in Volts, Current in Amperes and Power in Watts.)

\begin{tabular}{|c|c|c|c|c|c|}
\hline & Parameters & & Conditions & Code(Before & Code(After \\
\hline Voltage & Current & Power & & Encoding) & Encoding) \\
\hline (in Volts) & (in Amperes) & (in Watts) & & & \\
\hline 230 & $0.26-0.27$ & 60.63 & ON/Normal & B & 0 \\
\hline 229.8 & $0.28-0.30$ & 64.71 & Load & c & 1 \\
\hline 230 & $0.31-0.33$ & $72-76$ & High Load & D & 2 \\
\hline \multirow[t]{3}{*}{229} & 0.33 and above & 77 and above & Extremely & E & 3 \\
\hline & & & High Load & & \\
\hline & & & & & \\
\hline
\end{tabular}

Step 5: Deployment of the Trained Model

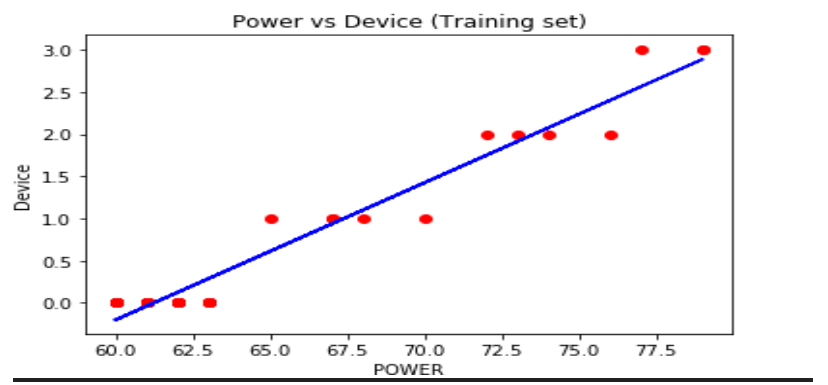

Figure 4.4: Training Result of Simple Linear Regression when Applied to Collected Dataset of Power for Motor 1. 


\section{International Journal of Engineering Applied Sciences and Technology, 2019 Vol. 4, Issue 4, ISSN No. 2455-2143, Pages 128-135 \\ Published Online August 2019 in IJEAST (http://www.ijeast.com)}

Figure 4.4 shows the graph of power verses device (single phase AC motor)for Training dataset. According to the above graph of figure 4.4 when power is in between 60-63 watts then according to Table 1 it represents ON/Normal condition. This condition is represented by code B before encoding, but as machine learning algorithm learns only numeric characters hence it is necessary to convert this alphabetic code into numeric code thus code B before encoding is represented as 0 after encoding. Here power is measured in Watts

\section{Step 6: Getting Predictions from the Existing Model}

Machine Learning uses data to answer the questions so prediction is that step where we finally get to recognize the answers for our questions. The power of ML is that we were able to determine the performance of our motor under test, failure of motor and remaining useful life of motor under test for predictive maintenance using our model rather than using human judgement and manual rules.

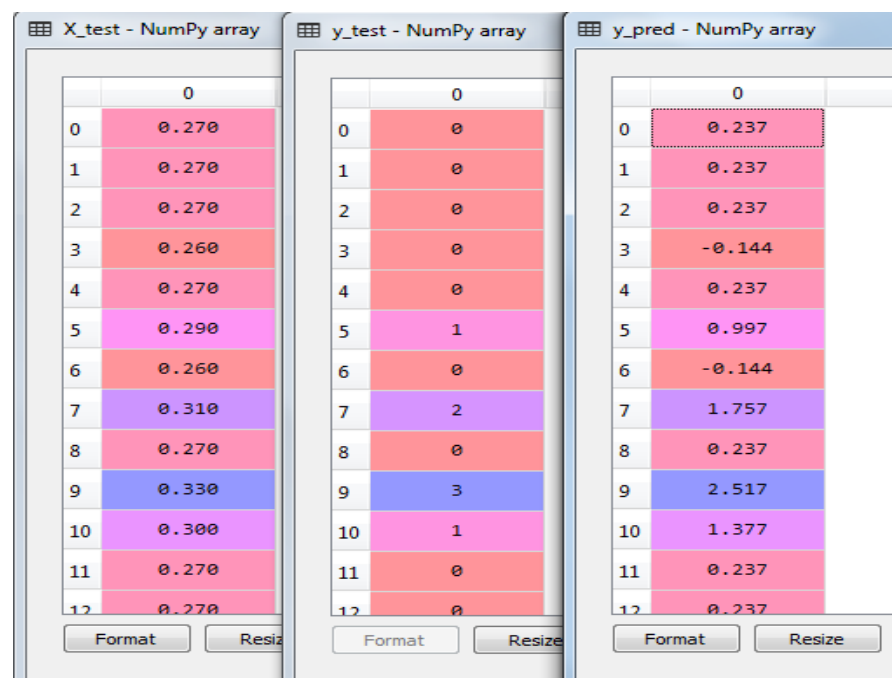

Figure 4.5: Comparison of Input Test Data, Output Test Data and Predicted Output Data when Simple Linear Regression

Algorithm is Applied to Collected Dataset of Current for Motor 1.

According to figure 4.5 and table 1 when current is between 0.28 to 0.30 it represents the Load condition at this time, code after encoding is 1 which is represented exactly 1 in above figure 4.5 and this condition is predicted as 0.997 which is approximately equals to 1 .

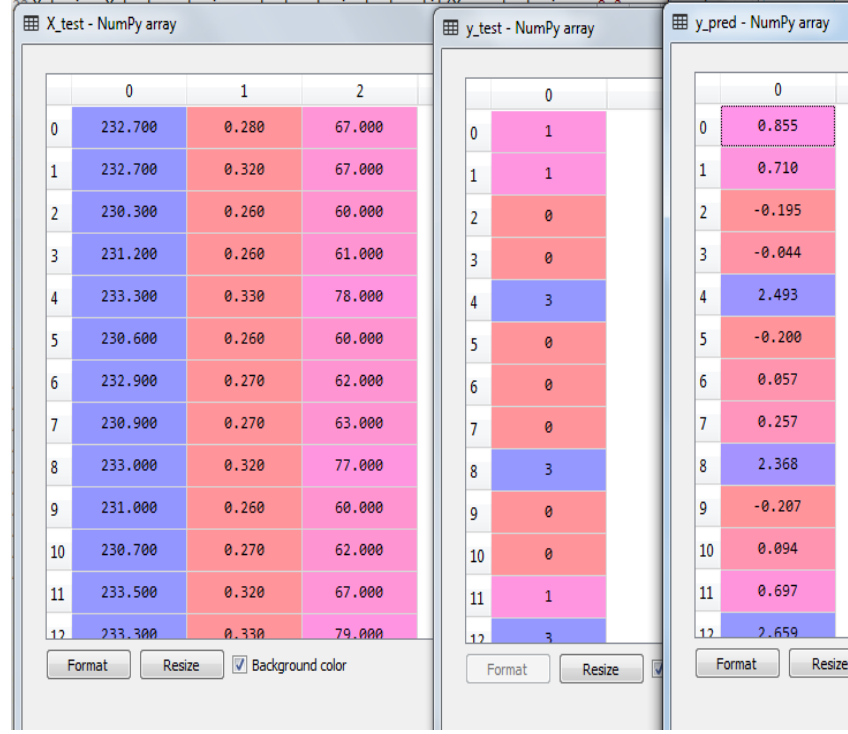

Figure 4.6: Comparison of Input Test Data, Output Test Data and Predicted Output Data when Multiple Linear Regression

Algorithm is applied to Collected Dataset for Motor 1.

In Multiple Linear Regression a single response variable $\mathrm{Y}$ which is a output variable depends linearly on a number of predictor variables that is input variables or key variables. It can be to find out which factor has the highest impact on the predicted output and vice versa and how different variables relate to each other. Code $\mathrm{B}$ before encoding is represented as 0 after encoding and it indicates the ON/Normal condition, Code $\mathrm{C}$ before encoding is represented as 1 after encoding and it indicates the Load condition which means that load is connected. Code D before encoding is represented as 2 after encoding and it indicates the High Load condition which means that load is connected but consumes more current which increases power consumption linearly. Code E before encoding is represented as 3 after encoding and it indicates the Extremely high load condition which means that load is connected but consumes much more current which increases power consumption linearly and it indicates that there is maintenance requirement for $\mathrm{AC}$ motor under test.

\section{CONCLUSION \& FUTURE SCOPE}

The foremost objective of predictive maintenance of machine is to predict when device failures can arise. Then prevent that failure by taking appropriate actions. By completing this work we are proficient to effectively calculate the voltage, current, and power related to motor under test, and also we are able to make predictions based on this collected data. Predictive Maintenance System monitors future failures and will schedule maintenance in advance. IoT based machine learning will help overcome major restrictions in productivity, efficiency and maintenance costs related with it. This existing 
system and tool sequence supports a growing IoT architecture. The supervised models can be used to find a clear and deep understanding of a complicated problem or situation from the data and the consequent use of prognostics and estimating will make definite that the production process runs efficiently with minimal costs incurred for maintenance and reduce product quality degradation. This results in numerous costs savings.

In future we can use different Machine Learning (ML) algorithms which will cover up more areas of industries, by using different algorithmic techniques with different type of machines and different production techniques with more advanced prediction. In future we can add more inventiveness in the existing system which can be implemented easily using different hardware and software and by using different Machine Learning techniques.

\section{REFERENCES}

[1] Jakra Husain, Ashish Manusmare (2019) -“Modelling of Industrial Machine Sturcture for Predictive Maintenance Using IoT Sensor Data and Machine Learning-A Review",IOSR-JECE, India, Mar.-Apr, Volume 14, Issue 2, Ser. II, (PP 16-21).

[2] "Predictive analytics and machine learning". https://www.sas.com/en_gb/insights/articles/analytics/aguide-to-predictive-analytics-and-machinelearning.html.

[3] Junmin Park, Hyunjae Park and Young-June Choi (2018) "Data Compression and Prediction Using Machine Learning for Industrial IoT",-The $32^{\text {nd }}$ International Conference on Information Networking (ICOIN 2018), IEEE, Suwon, Republic of Korea, (pp. 818-820).

[4] Ameeth Kanawaday, Aditya Sane (2017) -"Machine Learning for Predictive Maintenance of Industrial Machines using IoT Sensor Data". IEEE, Pune, India, (pp. 87-90).

[5] Mr.Purnendu Shekhar Pandey (2018) "Machine Learning and IoT for Prediction and Detection of Stress", IEEE, Haryana, India.

[6] Alejandro Correa Bahnsen, Djamila Aouada, Bjorn Ottersten (2015) "Ensemble of Example-Dependent Cost-Sensitive Decision Trees", Elsevier, University of Luxembourg, Luxembourg.

[7] Susto, G. A., Schirru, A., Pampuri, S., McLoone, S., \& Beghi, A. (2015) "Machine Learning for Predictive Maintenance: A Multiple Classifiers Approach". IEEE Transactions on Industrial Informatics, 11(3), (pp. 812820). 